\title{
EVENT-TRIGGERED REAL-TIME METERING IN SMART GRIDS
}

\author{
Stefan Werner and Jarmo Lundén
}

\author{
Aalto University School of Electrical Engineering \\ Department of Signal Processing and Acoustics, Finland \\ Email: \{stefan.werner, jarmo.lunden\}@aalto.fi
}

\begin{abstract}
This paper introduces an event-triggered approach to realtime metering that significantly reduces the amount of reported measurements. The proposed method reports measurements only when the load profile changes due to a triggering event, and ensures that the maximum difference between reported and true measurement values is always bounded in magnitude. Moreover, it employs a change detection based filter to resolve the estimation-tracking conflict associated with non-stationary signals containing abrupt changes. Simulation results show that the proposed reporting method provides a convenient tradeoff between average reporting error as well as the reporting frequency.
\end{abstract}

Index Terms - Change detection, load profile, eventtriggered communication, smart grid, smart metering.

\section{INTRODUCTION}

The future electric power grid has to be supported by a sensing and communication infrastructure capable of providing situational awareness [1,2]. Smart meters are prime examples of such infrastructure. Smart meters collect and report real-time load measurements from the end-users in the distribution grid to a central processing point (CPP). To capture the dynamics most measurement and communication sensors have been calibrated to measure and transmit at an electronic time scale of several hundred times per second [3], while significant changes in the load profile happen at a much longer time scale. Thus, continuously reporting measurements will waste valuable resources such as bandwidth and energy, as well as result in storing large data sets containing relatively few informative data values. Consequently, an approach that is more selective in its measurement reporting would be attractive.

One obvious approach is to periodically report compressed data obtained by, e.g., averaging over a fixed time window. This approach is sufficient when detailed knowledge of the actual consumption pattern is not important, e.g., for billing purposes. However, whenever reported measurements are used for reconstructing or predicting load profiles, the use of averaged data may lead to large errors and increased latency. In particular, abrupt changes due to on/off events can cause large instantaneous errors [4] between reported and actual values. Therefore, any developed selective reporting scheme should react quickly to abrupt changes in the load profile to ensure an accurate load reconstruction at the CPP.

A number of papers has been published that focus on reducing the communication rate by only sending measurements whenever a significant event has occurred [3,5-8]. In particular, a measurement is transmitted only if it differs by more than a predefined threshold from the previous measurement. The basic underlying idea is that the load profiles of all household appliances are piecewise constant, i.e., they follow the so-called on/off model [9]. In other words, an appliance that is switched on or off will cause an abrupt change in the load profile, and the power consumption is approximately constant between changes.

The typical load profiles are much more complicated than the simple on/off model. The above thresholding approach only works for abrupt changes that exceed the bound. In fact, it is incapable of identifying a slowly growing or decaying load profile, which is commonly seen in practice, see, e.g., [9-13]. As a result, the last reported measurement value may differ considerably from the current load. Consequently, when designing a method that reduces the amount of data transfer it is of paramount importance to also ensure that the reporting error is bounded. In [14], we proposed a Shewhart change detection based reporting scheme that guarantees that the worst-case error is upper bounded by a predefined user controlled threshold. In this paper, we extend the reporting scheme in [14] by proposing a reporting scheme that not only reacts to abrupt changes and significant drifts, e.g., due to turning an appliance on or off, but also to smaller events which enables better tracking of appliances' power consumption.

This paper introduces a double-threshold load tracking and reporting (DT-LTR) scheme. The DT-LTR scheme employs a change detection based filter for tracking of the local mean trend. The change detection filter employs a threshold to reset the tracking in response to abrupt changes in the load profile. This threshold ensures that the worst-case error is upper bounded. In addition, a second threshold is introduced to control the reporting accuracy of the local mean 
estimate at the CPP. Our simulation results with real measurement data, show that the proposed reporting scheme reduces the reporting requirements of real-time metering significantly in exchange for a minor tracking error at the CPP.

\section{EVENT-TRIGGERED REAL-TIME METERING}

In this section, we propose and analyze a double-threshold load tracking and reporting (DT-LTR) scheme that works with one-second or sub-second time interval sampling. The DTLTR scheme extends the Shewhart-test based reporting (STR) scheme in [14], and features accurate load tracking and reduced reporting rate. We note that the actual measured and transmitted quantity may be either real or reactive power. In the following, we will refer to this quantity simply as load, denoted by $x_{t}$, regardless of whether it is real or reactive power. Next we briefly summarize the STR scheme which serves as a motivation for the introduction of DT-LTR scheme.

\subsection{Shewhart test based reporting (STR) scheme}

The basic idea behind the STR scheme is to address one characteristic aspect commonly observed in typical household and appliance load curves. First, when an appliance is turned on or off, what typically follows is a rapid increase or decrease in electricity consumption. These quick changes, which can be in the order of several kilowatts, have to be detected and reported as quickly as possible. This was accomplished by the Shewhart test in [14]. The STR scheme transmits measurements $x_{t}$ only when the load profile changes due to a triggering event as follows

$$
\text { Report } x_{t} \text { if }\left|x_{t}-\mu_{t-1}\right|>\lambda
$$

where $\mu_{t-1}$ denotes the last reported load value, which serves as the load estimate at the remote CPP. This reporting rule ensures that the maximum error between the measurement and the load estimate at the CPP is always bounded in magnitude by $\lambda$.

\subsection{Double threshold load tracking and reporting}

The STR scheme is good for detecting large changes quickly. In fact, any change larger than the threshold will be detected with one observation. In addition, gradual drifts exceeding $\lambda$ will be always detected, as opposed to the approaches in $[3,5,7]$. However, the Shewhart test is not capable of detecting small changes in which the load variation around $\mu_{t}$ stays within $\pm \lambda$. And, although the Shewhart test guarantees a bounded error, even a small instantaneous error persisting over a long period of time can result in a large cumulative error over time. This is the aspect that we want our algorithm to address and where the double-threshold load tracking and reporting scheme comes into play.
Instead of using instantaneous measurement values as the basis for selective reporting, the proposed DT-LTR scheme employs an estimator for tracking the mean trend of the local load profile and uses the estimated trend to decide whether to report. Let $s_{t}$ denote the trend and $\hat{s}_{t}$ its estimate. In order to account for abrupt and gradual changes in $s_{t}$, the DT-LTR scheme estimates $s_{t}$ recursively as

$$
\hat{s}_{t}= \begin{cases}\hat{s}_{t-1}+\alpha \epsilon_{t} & \text { if }\left|x_{t}-\mu_{t-1}\right| \leq \lambda \\ x_{t} & \text { otherwise }\end{cases}
$$

where $\epsilon_{t}=x_{t}-\hat{s}_{t-1}$ is the a priori estimation error and $0<\alpha \leq 1$ is a step size that controls the estimation accuracy. Threshold $\lambda$ forces estimator $\hat{s}_{t}$ to reset whenever an abrupt change in the load profile takes place.

The DT-LTR scheme guarantees that the difference between the local trend estimate and the load estimate at the CPP is bounded in magnitude by a predefined threshold, i.e., $\left|\hat{s}_{t}-\mu_{t}\right| \leq \gamma$. Consequently, $\hat{s}_{t}$ is reported as follows

$$
\text { Report } \hat{s}_{t} \text { if }\left|\hat{s}_{t}-\mu_{t-1}\right|>\gamma
$$

where threshold $\gamma \leq \lambda$ allows for a trade off between the reporting frequency and the error $\hat{s}_{t}-\mu_{t}$. If $\hat{s}_{t}$ is not reported, the CPP will use $\mu_{t}=\mu_{t-1}$ as the current load estimate.

The DT-LTR scheme is summarized in Algorithm 1. Note that for $\alpha=1$ and $\gamma=\lambda$, the DT-LTR scheme reduces to the STR scheme in (1). Moreover, the DT-LTR scheme resembles a hybrid version of the classic Shewhart [15] and the exponentially weighted moving average (EWMA) [16] charts, which has been considered for general change detection and control problems, e.g., in [17] and [18]. We note that in addition to the difference in the underlying philosophy of the application at hand and that of control charts, the DT-LTR scheme resets estimator $\hat{s}_{t}$ according to (3) which enables better tracking between abrupt changes.

The choice of step size $\alpha$ in (2) affects both the variance and the memory of estimator $\hat{s}_{t}$. In particular, assuming that the measurements follow an additive model

$$
x_{t}=s_{t}+e_{t},
$$

where the disturbance sequence $e_{t}$ is independent and identically distributed, then for a sufficiently large threshold $\lambda$ we have $E\left[x_{t}\right]=s_{t}$ and $\sigma_{x}^{2}=\sigma_{e}^{2}$. In this case, $\hat{s}_{t}$ is an unbiased estimator of a constant load $s_{t}$, i.e., $E\left[\hat{s}_{t}\right]=s_{t}$, with variance

$$
\sigma_{\hat{s}_{t}}^{2}=\frac{\alpha+(1-\alpha)^{2 t+1}}{2-\alpha} \sigma_{x}^{2} .
$$

Under the same assumptions, the effective window length (memory) of estimator $\hat{s}_{t}$, denoted by $N_{\text {eff }}$, can be defined as the number of samples required for $\sigma_{\hat{s}_{t}}^{2}$ in (5) to equal the variance of the sample mean estimator:

$$
N_{\text {eff }}=\lim _{t \rightarrow \infty} \frac{2-\alpha}{\alpha+(1-\alpha)^{2 t+1}}=2 \alpha^{-1}-1 .
$$




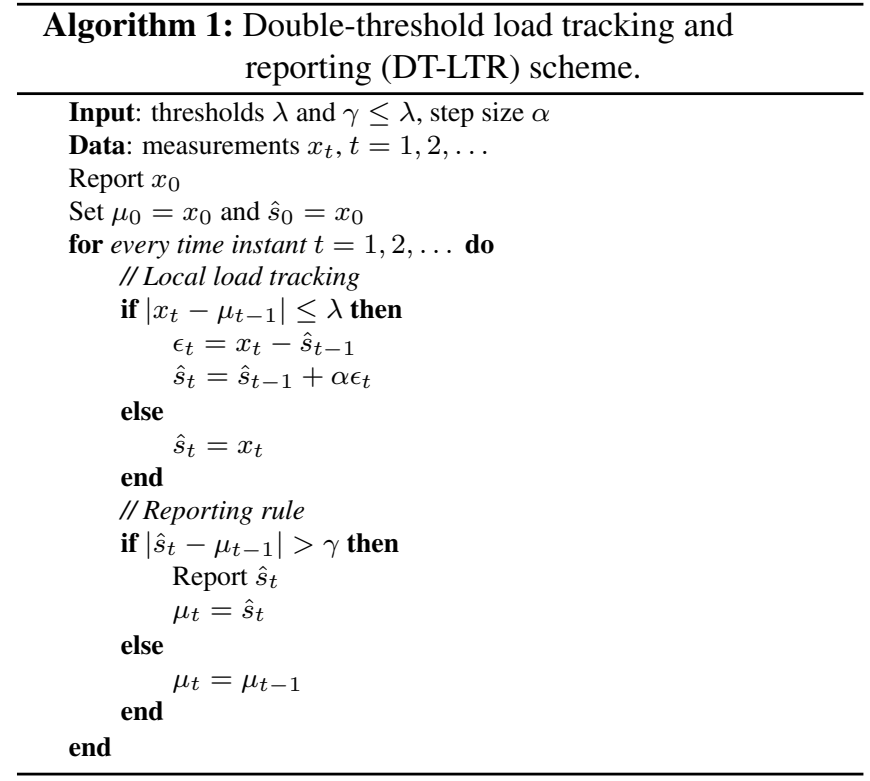

Remark 1. From (5) and (6) it follows that, for a fixed threshold $\gamma$, the reporting frequency of the DT-LTR scheme increases (decreases) when $\alpha$ increases (decreases).

Remark 2. The DT-LTR scheme allows the error $\left|x_{t}-\mu_{t}\right|$ to occasionally exceed $\gamma$ and be in the interval $[\gamma, \lambda)$. However, from (5) it follows that for most cases $\left|x_{t}-\mu_{t}\right| \leq \gamma$ whenever threshold $\gamma$ is set to a value larger than $\sigma_{x}$.

\section{SIMULATION RESULTS}

In this section, we compare the proposed load reporting scheme to the STR scheme [14] and the reduced-reporting scheme advocated in $[3,5,7]$, here referred to as finite difference reporting (FDR), using real measurements from the Smart* load dataset [19]. The Smart* dataset includes real power measurements with one-second sampling interval for three different houses over several months. Here, we consider Home B, a two-story house with eight rooms and four full-time occupants [19].

Fig. 1 shows a comparison of the reporting rates of the different reporting schemes for the month of May. Fig. 1 (a) plots the mean absolute error $E\left[\left|x_{t}-\mu_{t}\right|\right]$ versus the reporting rate. The proposed DT-LTR scheme achieves the lowest average error for all compared reporting rates. The second best is the STR reporting scheme while the FDR scheme is always significantly worse than the other two schemes. Fig. 1 (b) compares the reporting rates of the STR and FDR schemes relative to the reporting rate of the DT-LTR scheme for the same average error. The FDR scheme results in significantly higher reporting rates than those of the DT-LTR scheme. When the desired average error is between 2 and $4 \mathrm{~W}$, the FDR scheme requires roughly five times higher reporting rate than the DT-LTR scheme requires for the same average

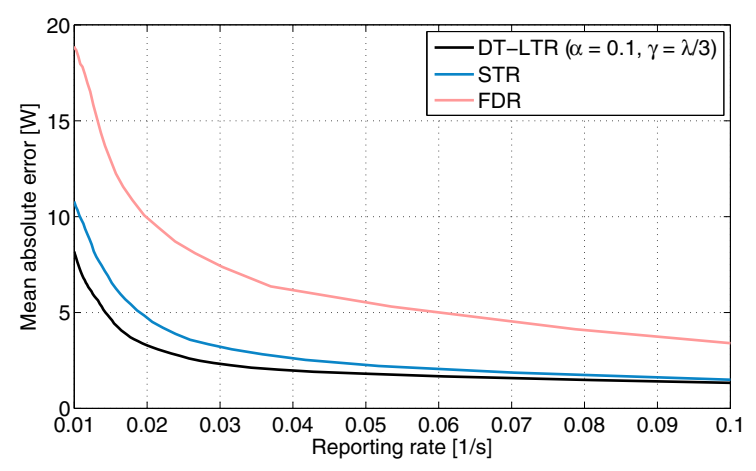

(a) Mean absolute error vs. reporting rate.

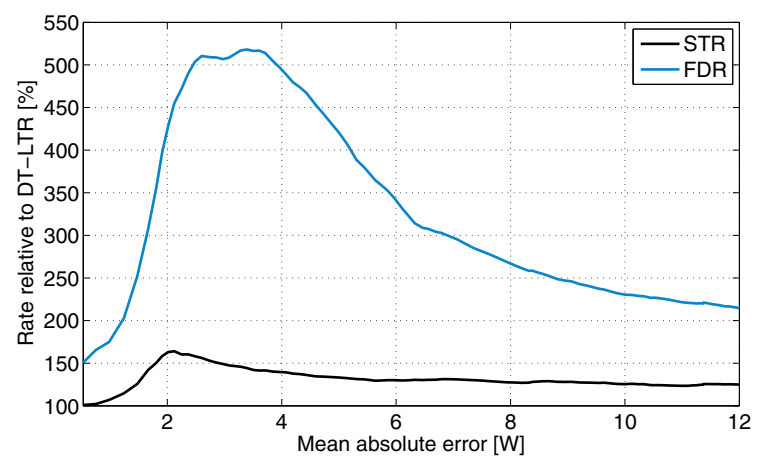

(b) Reporting rate relative to the rate of DT-LTR vs. mean absolute error.

Fig. 1. Comparison of the reporting rates vs. the mean absolute error for the Smart* dataset's [19] Home B load in May.

error. The STR scheme performs considerably better than the FDR scheme. However, the required increase in the reporting rate compared to the reporting rate of the DT-LTR scheme is over $60 \%$ for a desired average error close to $2 \mathrm{~W}$ and then reduces to a $25 \%$ increase as the desired average error increases. For very small average errors the reporting rate of the STR scheme is roughly the same as the reporting rate of the DT-LTR scheme.

Fig. 2 depicts the mean and $99 \%$ confidence interval of the absolute error $\left|x_{t}-\mu_{t}\right|$ at the CPP as a function of the test thresholds. Note that the y-axes are different in the different subfigures. Both the STR and DT-LTR scheme guarantee that the error is always upper bounded by the test threshold $\lambda$. The DT-LTR scheme has lower average error for the same upper bound $\lambda$ due to the improved EWMA-based local load tracking and the reporting associated to it. The FDR scheme, on the other hand, does not guarantee an upper bounded error. Fig. 2 (c) illustrates that the error can significantly exceed the test threshold $\lambda$, e.g., for $\lambda=100 \mathrm{~W}$ the error exceeds $300 \mathrm{~W}$ over $1 \%$ of the time. In addition, the mean absolute error is considerably larger as well. 


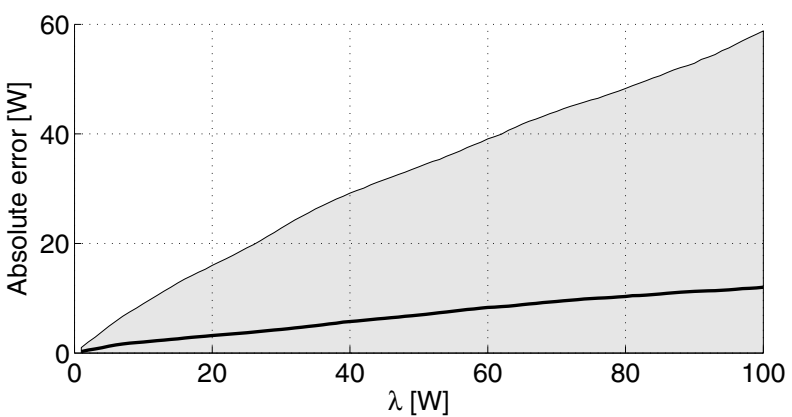

(a) DT-LTR

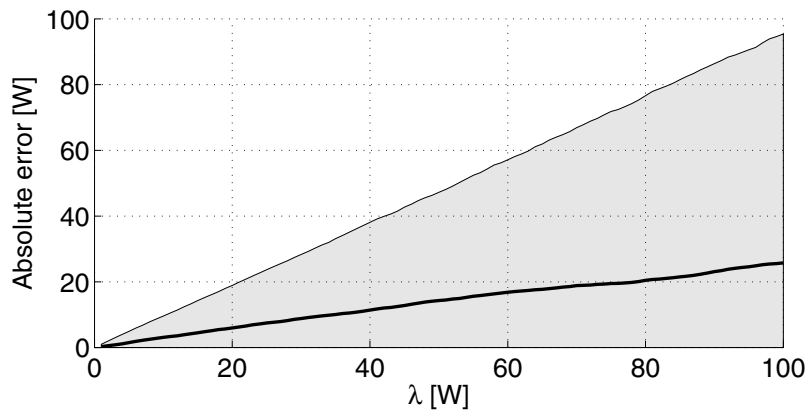

(b) STR

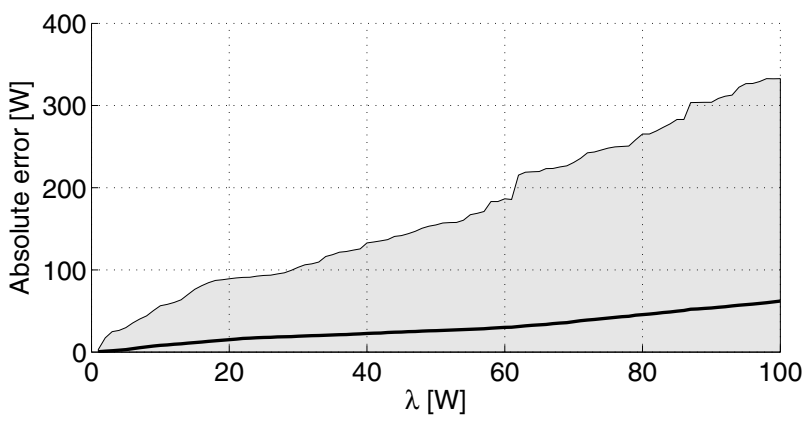

(c) FDR

Fig. 2. Mean and $99 \%$ confidence interval for the absolute error $\left|x_{t}-\mu_{t}\right|$ at the CPP as a function of the test threshold $\lambda$ for the Smart* dataset's [19] Home B load in May.

\section{CONCLUSIONS}

This paper proposed a double-threshold change-detection based filter for real-time metering to enable accurate load tracking and reduced reporting frequency. The proposed approach can track both abrupt changes and gradual drifts while still ensuring that the tracking error at the receiving end is always upper bounded. Our simulation results with real measurement data show that the proposed reporting scheme reduces the communication requirements significantly in exchange for a small tracking error at the receiving end.

\section{REFERENCES}

[1] Y.-H. Huang, S. Werner, J. Huang, N. Kashyap, and V. Gupta, "State estimation in electric power grids meeting new challenges presented by the requirements of the future grid," IEEE Signal Processing Magazine, vol. 29, no. 5, pp. 33-43, Sep. 2012.

[2] N. Kashyap, S. Werner, Y.-H. Huang, and T. Riihonen, "Power system state estimation under incomplete PMU observability - a reduced-order approach," IEEE Journal of Selected Topics in Signal Processing, vol. 8, no. 6, pp. 1051-1062, Dec. 2014.

[3] K. Armel, A. Gupta, G. Shrimali, and A. Albert, "Is disaggregation the holy grail of energy efficiency? The case of electricity," Energy Policy, vol. 52, no. C, pp. 213-234, Jan. 2013.

[4] A. Wright and S. Firth, "The nature of domestic electricity-loads and effects of time averaging on statistics and on-site generation calculations," Applied Energy, vol. 84, no. 4, pp. 389-403, Apr. 2007.

[5] H. Li, S. Gong, L. Lai, Z. Han, R. Qiu, and D. Yang, "Efficient and secure wireless communications for advanced metering infrastructure in smart grids," IEEE Transactions on Smart Grid, vol. 3, no. 3, pp. 15401551, Sep. 2012.

[6] M. Simonov, "Event-driven communication in smart grid," IEEE Communications Letters, vol. 17, no. 6, pp. 1061-1064, Jun. 2013.

[7] K. Samarakoon, J. Ekanayake, and N. Jenkins, "Reporting available demand response," IEEE Transactions on Smart Grid, vol. 4, no. 4, pp. 1842-1851, Dec. 2013.

[8] M. Simonov, "Hybrid scheme of electricity metering in smart grid," IEEE Systems Journal, vol. 8, no. 2, pp. 422-429, Jun. 2014.

[9] G. W. Hart, "Nonintrusive appliance load monitoring," Proceedings of the IEEE, vol. 80, no. 12, pp. 18701891, Dec. 1992.

[10] S. W. Lai, G. G. Messier, H. Zareipour, and C. H. Wai, "Wireless network performance for residential demandside participation," in Proc. IEEE Int. Conf. Innovative Smart Grid Technologies Europe, Göteborg, Sweden, Oct. 2010, pp. 1-4.

[11] Z. Wang and Z. Guilin, "Residential appliances identification and monitoring by nonintrusive load method," IEEE Transactions on Smart Grid, vol. 3, no. 1, pp. 8092, Mar. 2012. 
[12] S. Barker, S. Kalra, D. Irwin, and P. Shenoy, "Empirical characterization and modeling of electrical loads in smart homes," in Proc. IEEE Int. Green Computing Conference, Arlington, VA, USA, Jun. 2013, pp. 1-10.

[13] M. Pipattanasomporn, M. Kuzlu, S. Rahman, and Y. Teklu, "Load profiles of selected major household appliances and their demand response opportunities," IEEE Transactions on Smart Grid, vol. 5, no. 2, pp. 742750, Mar. 2014.

[14] J. Lundén and Werner, "Real-time smart metering with reduced communication and bounded error," in Proc. IEEE Int. Conf. Smart Grid Communications, Venice, Italy, November 3-6 2014, pp. 1-6.

[15] W. A. Shewhart, Economic Control of Quality of Manufactured Product, New York: American Society for Quality Control, 1980.
[16] S. W. Roberts, "Control chart tests based on geometric moving averages," Technometrics, vol. 1, no. 3, pp. 239250, Aug. 1959.

[17] J. M. Lucas and M. S. Saccucci, "Exponentially weighted moving average control schemes: Properties and enhancements," Technometrics, vol. 32, no. 1, pp. 1-12, Feb. 1990.

[18] G. Capizzi and G. Masarotto, "Evaluation of the runlength distribution for a combined Shewhart-EWMA control chart," Statistics and Computing, vol. 20, no. 1, pp. 23-33, Jan. 2010.

[19] S. Barker, A. Mishra, D. Irwin, E. Cecchet, P. Shenoy, and J. Albrecht, "Smart*: An open data set and tools for enabling research in sustainable homes," in Proc. 2012 Workshop Data Mining Applications in Sustainability, Beijing, China, Aug. 122012. 